\title{
Mg-Sb (Magnesium-Antimony)
}

\section{H. Okamoto}

The $\mathrm{Mg}$-Sb phase diagram in [Massalski2] was redrawn from [1984Nay].

[1990Oka] pointed out an unlikely feature in this phase diagram, i.e., the metastable melting point of $\alpha \mathrm{Mg}_{3} \mathrm{Sb}_{2}$ cannot be defined uniquely when extrapolated from the liquidus curves on the Mg-rich and Sb-rich side unless thermodynamically unlikely sudden change of curvature is introduced in these liquidus curves.

Figure 1 shows the $\mathrm{Mg}-\mathrm{Sb}$ phase diagram calculated by [2009Pal]. Naturally, the problem described above is solved in this figure.

Table 1 shows $\mathrm{Mg}-\mathrm{Sb}$ crystal structure data.

\section{References}

1984Nay: A.A. Nayeb-Hashemi and J.B. Clark, The Mg-Sb (Magnesium-Antimony) System, Bull. Alloy Phase Diagr., 1984, 5(6), p 579-584

1990Oka: H. Okamoto and T.B. Massalski, Thermodynamically Improbable Phase Diagrams, J. Phase Equilib. Diffus., 1990, 12(2), p 148-168

2009Pal: M. Paliwal and I.H. Jung, Thermodynamic Modeling of the $\mathrm{Mg}-\mathrm{Bi}$ and $\mathrm{Mg}-\mathrm{Sb}$ Binary Systems and Short-RangeOrdering Behavior of the Liquid Solutions, Calphad, 2009, 33, p 744-754

Table 1 Mg-Sb crystal structure data

\begin{tabular}{lccccc}
\hline Phase & Composition, at.\% Sb & Pearson symbol & Space group & Strukturbericht designation & Prototype \\
\hline$(\mathrm{Mg})$ & 0 & $h P 2$ & $P 6_{3} / m m c$ & $A 3$ & $\mathrm{Mg}$ \\
$\beta \mathrm{Mg}_{3} \mathrm{Sb}_{2}$ & $38.1-40$ & $c l 80$ & $I a \overline{3}$ & $D 5_{3}$ & $\mathrm{Mn}_{2} \mathrm{O}_{3}$ \\
$\alpha \mathrm{Mg}_{3} \mathrm{Sb}_{2}$ & $37.9-40$ & $h P 5$ & $P \overline{3} m 1$ & $D 5_{2}$ & $\mathrm{La}_{2} \mathrm{O}_{3}$ \\
$(\mathrm{Sb})$ & 100 & $h R 2$ & $R \overline{3} m$ & $A 7$ & $\alpha A s$ \\
\hline
\end{tabular}

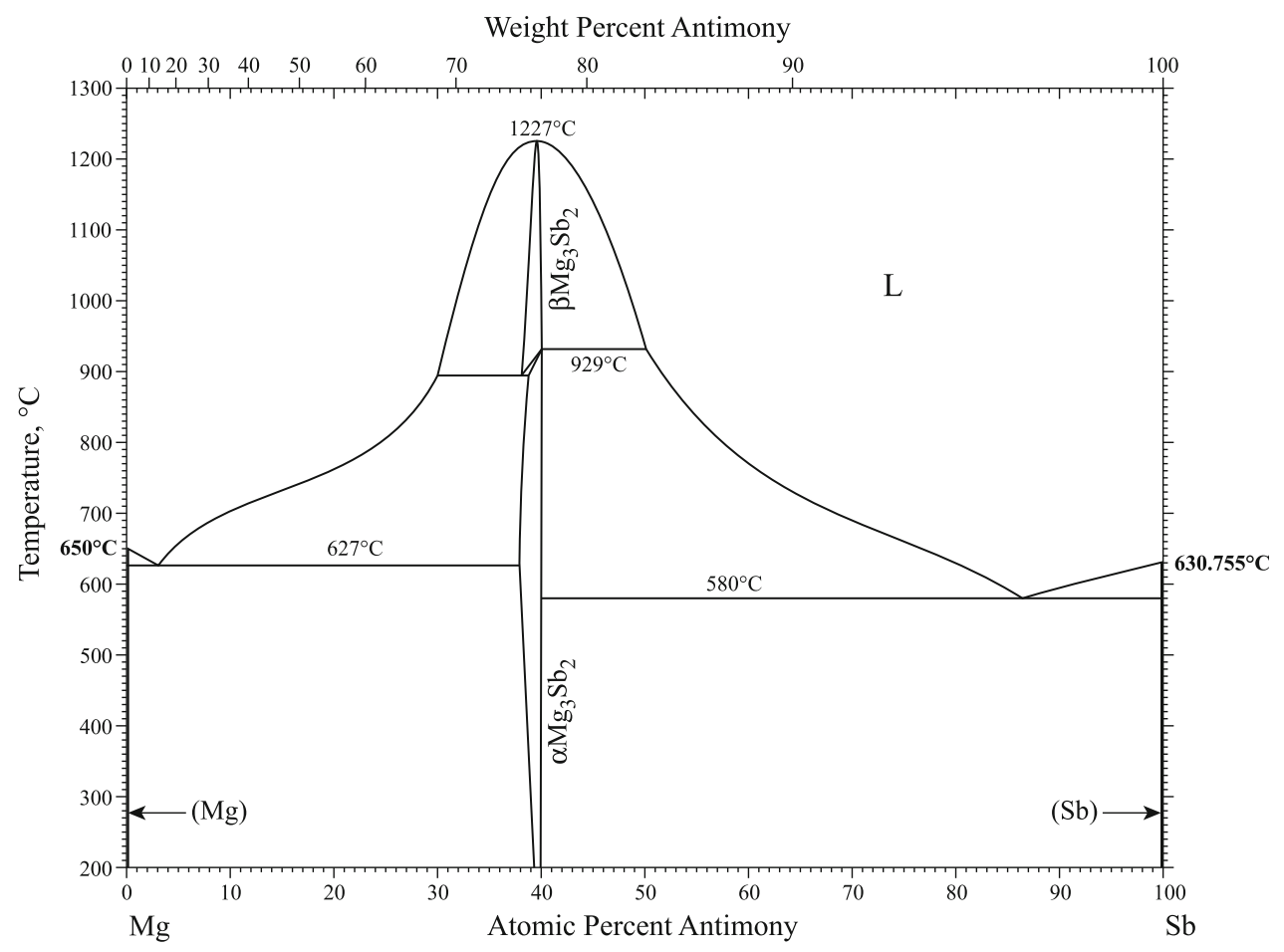

Fig. $1 \mathrm{Mg}$-Sb phase diagram 